Applied Physics A manuscript No.

(will be inserted by the editor)

\title{
X-ray magneto-optics of lanthanide materials: principles and applications
}

\author{
J.E. Prieto, O. Krupin, K. Döbrich, F. Heigl, G. Kaindl, K. Starke \\ Freie Universität Berlin, Arnimallee 14, D-14195 Berlin, Germany \\ Received: date / Revised version: date
}

\begin{abstract}
Lanthanide metals are a particular class of magnetic materials in which the magnetic moments are carried mainly by the localized electrons of the $4 f$ shell. They are frequently found in technically relevant sys' tems, to achieve, e.g., high magnetic anisotropy. Magnetooptical methods in the x-ray range are well suited to study complex magnetic materials in an element-specific way. In this work, we report on recent progress on the quantitative determination of magneto-optical constants of several lanthanides in the soft x-ray region and we show some examples of applications of magneto-optics to hard-magnetic interfaces and exchange-coupled layered structures containing lanthanide elements.
\end{abstract}

\section{Introduction}

The negligible overlap between the partially filled $4 f$ shells of neighboring atoms in lanthanide metals leads to strongly localized magnetic moments, which in general contain both an orbital and a spin part. The induced valence-band polarization gives only a minor contribution to the magnetization, in contrast to the predominantly itinerant moments of ferromagnetic transition metals (TM). The localized character of the $4 f$ moments is also responsible for the negligibly small direct exchange interaction between lanthanide ions. Instead, they couple only indirectly through the valence-band electrons (RKKY interaction), which leads to ordering temperatures typically below room temperature (RT) in lanthanide metals. Non-vanishing orbital moments give rise to non-spherical charge distributions in the $4 f$ shell, which lead to strong "single-ion" contributions to the magnetic anisotropy. In fact, the hardest magnetic materials known today are intermetallic systems containig both RE and TM ions as in Co-Sm and Nd-Fe-B. In these compounds, the high magnetic anisotropies are induced by the RE ions, while the characteristic high ordering temperatures of the ferromagnetic TMs are retained. [1]
Techniques based on magneto-optical (MO) effects in the visible-light region are widely used for the analysis of magnetic materials. 2] The MO Kerr effect (MOKE) finds technological applications as well in the reading process of MO disks. 3. MOKE is based on the difference in reflectivity of polarized light upon reversal of the local magnetization direction. Although MO effects are generally small in the visible-light region, sensitive detection methods (lock-in techniques) yield enough contrast to allow, e.g., the observation of domain structures in optical microscopy. 4] One powerful feature of MO techniques is their capability to monitor magnetization reversal processes in external magnetic fields. MO experiments in the visible-light region typically involve optical transitions between delocalized valence states. Therefore, except in special cases, it is rather difficult to separate the magnetic contributions of different elements.

Element selectivity has become a key issue in the analysis of magnetic nanostructures [5] 6] or heteromagnetic systems for information storage. 3. 7. It is naturally achieved by employing optical transitions that involve core electrons, i.e. by tuning the photon energy to absorption thresholds in the $\mathrm{x}$-ray range. The largest $\mathrm{MO}$ effects are found in transitions into electronic levels of partially occupied shells which contribute to the magnetic moment. These are the $L_{2,3}(2 p \rightarrow 3 d)$ absorption edges of the transition metals and the $M_{4,5}(3 d \rightarrow 4 f)$ and $N_{4,5}(4 d \rightarrow 4 f)$ thresholds of lanthanides. All these transitions occur in the soft $\mathrm{x}$-ray region.

It has been shown by several studies [8, 10] that in order to extract useful quantitative information from soft $\mathrm{x}$-ray MO signals in layered systems (e.g. layer-resolved magnetization profiles), comparisons with model calculations [11] of reflected intensities are required. Because the wavelength of soft $\mathrm{x}$-rays is often comparable with the dimensions of thin films and multilayers in the nanometer range, one has to consider interference effects. For their treatment in model calculations accurate values of the MO constants of the magnetic elements are required. While several experimental determinations of 
J.E. Prieto et al.

soft x-ray MO constants 12, 13, 14, 15,16 and reflection coefficients [17] have been reported for the $L_{2,3}$ thresholds of TMs, results on lanthanide elements have been scarce. Hence, we set out to experimentally determine the MO constants of lanthanide metals in the soft x-ray resonance regions.

The paper is organized as follows. After giving a short account of the experimental methods used, we describe the determination of the soft x-ray MO constants of Gd and Tb. We present then several examples of applications of the constants as input for calculations of MO properties of thin lanthanide films (magnetizationdependent reflectivity and Faraday rotation). Finally, two examples of using MO effects to study epitaxial lanthanide systems (the Sm/Co interface and Gd/Y/Tb trilayers) are discussed.

\section{Experimental}

Absorption experiments at the $4 d \rightarrow 4 f$ absorption thresholds were performed at UE56 undulator beamlines [18] of the Berliner Elektronenspeicherring für Synchrotronstrahlung (BESSY II), while those at the $3 d \rightarrow 4 f$ thresholds were performed at beamline ID12-B/HELIOS-I of the European Synchrotron Radiation Facility (ESRF). 19. 20. In the experiments at UE56, the photon energy resolution was set to about $100 \mathrm{meV}$ (full width at half maximum), which is well below the intrinsic width of the narrow $N_{4,5}$ pre-edge absorption lines of Gd and Tb. 21] By scanning the photon energy with a synchronized movement of monochromator and undulator, an easy normalization of the spectra was made possible. For the absorption measurements at the $M_{4,5}$ thresholds at ID12, the energy resolution was set to about $0.4 \mathrm{eV}$.

Absorption spectra were recorded in total-electron yield (TEY) mode using a high-current channeltron. To suppress the background of secondary electrons from the chamber walls, both the sample and a retarding grid in front of the channeltron were biased using a low-voltage battery. For signal stability, high voltage was supplied to the channeltron by a $3.2-\mathrm{kV}$ battery box. The electronyield current was amplified by an electrometer. All xray reflectivity measurements were performed at BESSY II. The specularly reflected $\mathrm{x}$-ray intensity was detected by a $\mathrm{Si}$ photodiode mounted on a rotation feedthrough inside the vacuum chamber.

Lanthanide-metal films have been prepared in situ by vapor deposition in ultra-high vacuum on a W(110) single-crystal substrate. External magnetic fields of up to $2 \mathrm{kOe}$ were applied using a rotatable electromagnet with a soft-iron yoke [22] in order to magnetize the films inplane along the substrate bcc[110] axis. This corresponds to the easy axis of Gd and Tb metal films. Further details on the preparation and characterization of RE films can be found in Ref. 23].

\section{MO constants}

\subsection{Absorption Coefficients}

Compared to transmission methods, which give directly the absorption coefficients, TEY detection has the advantage to allow the use of metallic single crystals as substrates, on which well-characterized epitaxial films can be grown. In particular, annealing of the deposited lanthanide films at the optimum temperatures results in smooth films with homogeneous thicknesses and high remanent magnetization. 24]
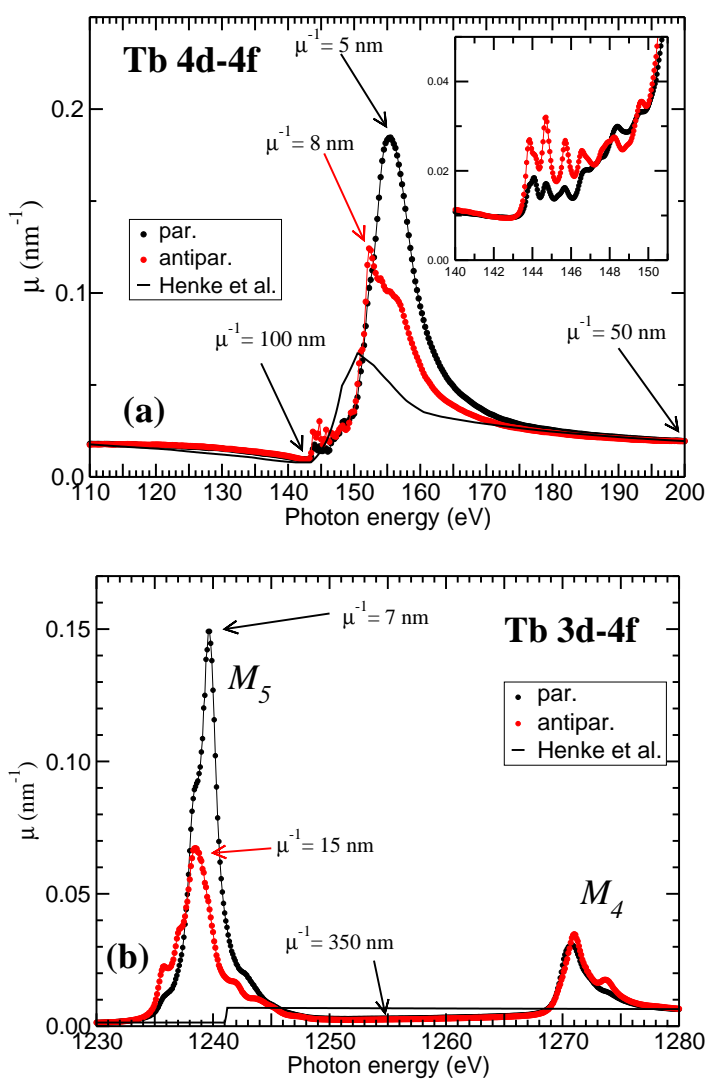

Fig. 1 Absorption coefficients of Tb metal at (a) the $4 d \rightarrow$ $4 f$ and (b) $3 d \rightarrow 4 f$ resonance regions for CP light propagating nearly parallel or antiparallel to the sample magnetization. Data of Henke et al. 25] are included for comparison. Values for of the light absorption length $\mu^{-1}$ are given for selected photon energies.

Figure 1 displays experimental absorption spectra in the $\mathrm{Tb} N_{4,5}(4 d \rightarrow 4 f)$ and $M_{4,5}(3 d \rightarrow 4 f)$ threshold regions with either nearly parallel or antiparallel orientation of magnetization and photon spin of the incoming circularly polarized (CP) light. The spectra have been scaled to match the tabulated values of Henke et al. [25] at the ends of the experimental photon energy ranges after having been corrected for intrinsic saturation. This affects the measured TEY spectra when the attenuation length of the electromagnetic radiation, $\mu^{-1}$, becomes 
comparable to $d_{e}$, the inelastic mean free path (IMFP) of electrons in the solid. 26 27] The saturation correction was performed using the relation given by van der Laan and Thole [27] and has been described in detail elsewhere 28.

Lanthanide absorption spectra at the $4 d \rightarrow 4 f$ excitation thresholds can be divided into two regimes, as shown in Fig. 1(a). The so-called pre-edge lines at low energies would be 'dipole-forbidden' in the hypothetical limit of strict Russel-Saunders coupling and their presence is only possible due to the $4 d$ spin-orbit interaction. Owing to small Auger matrix elements, these pre-edge lines have narrow life-time widths of some $350 \mathrm{meV}$ [21]. In contrast, the large absorption maxima (giant resonances) comprise several strong and broad absorption lines. The characteristic asymmetric shape ('Beutler-Fano' profile) is due to the quantum-mechanical interference of two excitation channels leading to the same final state: the direct photoemission from the $4 f$ level into the continuum and the absorption from the $4 d$ into the $4 f$ shell followed by a rapid super-Coster-Kronig decay. Since both channels have similar probabilities at the lanthanide $N_{4,5}$ thresholds (as revealed by a small Fano $q$ parameter of about $3[23]$ ), the resonance shape is highly asymmetric; this in particular leads to a strong variation of the light absorption length across the giant resonance where values range from a few nanometers at the maxima to hundred nanometers at the antiresonance region, as shown in Fig. 1(a).

At the lanthanide $M_{4,5}$ thresholds $(3 d \rightarrow 4 f$ transitions), the photoemission channel is much weaker than the absorption channel so that the Fano $q$ parameters are of the order of 100. 23] For such large $q$ values, the Fano profile approaches the Lorentzian shape and, in fact, the $M_{4,5}$ absorption spectra contain hundreds of Lorentzianshaped multiplet components. The spin-orbit coupling in the $3 d$ shell is the strongest interaction in the final state, so that the components cluster into two groups, the $M_{5}$ and $M_{4}$ thresholds, 29] depending on whether the spin of the $3 d$ hole state is oriented parallel or antiparallel to the $l=2$ orbital angular momentum, respectively. As for the $N_{4,5}$ thresholds, the absorption lengths change dramatically as the photon energy is scanned through the $M_{4,5}$ resonances (see Fig. 1(b)).

From the values obtained for the absorption coefficients (Fig. 1) we are able to calculate the magnetooptical constants, i.e. the real and imaginary parts of the complex index of refraction, defined as: $25 n_{ \pm}(E)=$ $1-\delta_{ \pm}(E)-i \beta_{ \pm}(E)$. The + and - signs refer to the magnetization pointing either parallel or antiparallel to the CP photon spin, respectively. The imaginary part is directly related to the absorption coefficient through $\beta_{ \pm}(E)=\frac{1}{4 \pi} \frac{h c}{E} \mu_{ \pm}(E)$, while the real parts can be calculated by means of a Hilbert transformation using the Kramers-Kronig relations. 28]

The calculated real parts of the refractive index of $\mathrm{Tb}$ at the $N_{4,5}$ and $M_{4,5}$ absorption thresholds are shown
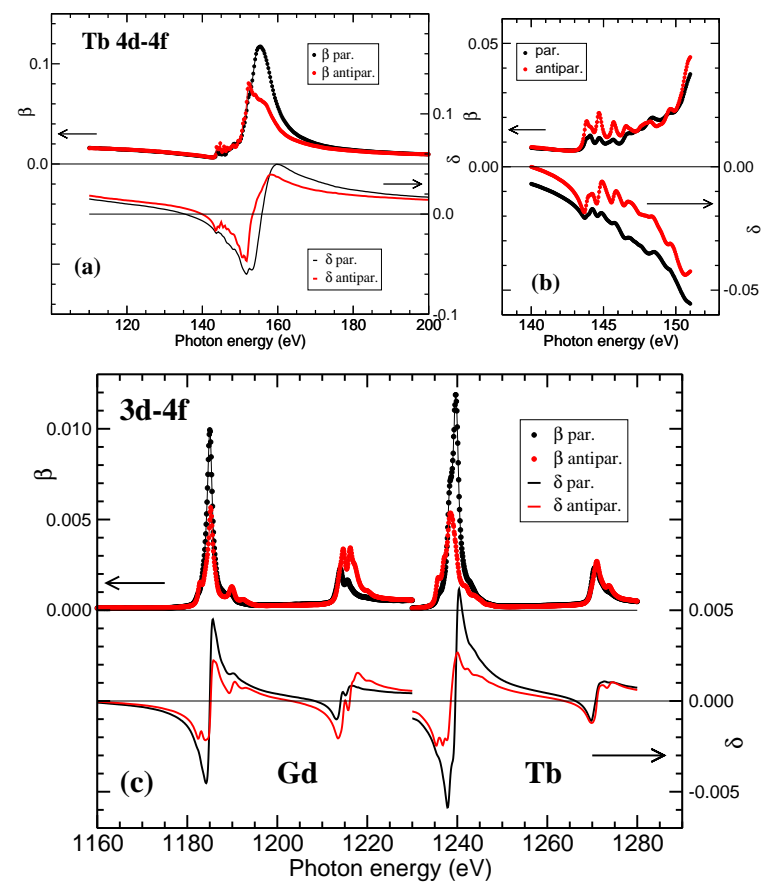

Fig. 2 MO constants of (a) $\mathrm{Tb}$ at the $4 d \rightarrow 4 f$; (b) the $\mathrm{Tb}$ $4 d \rightarrow 4 f$ pre-resonance structure, and (c) Gd and $\mathrm{Tb}$ at the 3d-4f threshold.

in Fig. 2 for opposite orientations of magnetization and photon spin. The spectra exhibit the well-known dispersive behaviour with significant tails reaching far below and above the peaks of the associated imaginary parts. While the magnetic contrast of the latter is found mainly in the absorption maxima, the real parts provide magnetic contrast also in regions where the absorption is small. Since the reflected signal is determined by both parts of the refractive index, this allows magnetizationdependent measurements in reflectivity with widely different penetration depths of the incoming light.

\section{Applications}

\subsection{Faraday rotation}

The MO constants can be applied in calculations of MO properties. As a first example we consider the Faraday effect. The rotation angle of linearly polarized (LP) light passing through a perpendicularly magnetized film is proportional to the difference $\Delta n=\left(\delta_{+}-\delta_{-}\right)$of the real parts of the refractive index for the two opposite light helicities into which LP light can be decomposed. The associated difference in the imaginary parts, $\left(\beta_{+}-\beta_{-}\right)$gives rise to ellipticity, i.e. the outcoming light is no longer linearly but elliptically polarized. The Faraday effect at $\mathrm{TM} \mathrm{L}_{2,3}$ edges has been studied in Refs. [14, 15, 16].

We have calculated the specific Faraday rotation (FR) and ellipticity for a thin Gd film at the $4 d \rightarrow 4 f$ thresh- 
old. The result is shown in Fig. 3] At $149 \mathrm{eV}$, the maximum of the giant resonance, the imaginary part of the refractive index does not depend on the magnetization direction, so that the ellipticity vanishes, and the FR reaches the huge value of $0.7^{\circ}$ per $\mathrm{ML}$ as a consequence of the large difference in the real parts of the refractive index $\left(\delta_{+}-\delta_{-}\right)$, which in turn is due to the large difference in absorption $\left(\beta_{+}-\beta_{-}\right)$. For comparison, the specific FR at the Fe $L_{2,3}$ edge is 10 times smaller. 14 An interesting open question is whether this huge value for the FR, as calculated here assuming a continuous medium, will also hold for ultrathin films of thicknesses in the range of a few monolayers.

We have proposed to use this large FR to build a "line switch", i.e., a device for fast switching between $s$ and $p$ polarization of soft x-rays at $h \nu=149 \mathrm{eV}$. 30 To this end, a $18.5 \mathrm{~nm}$ Gd film would be required in order to achieve a $90^{\circ}$ rotation of the light polarization plane upon reversal of the film magnetization direction. We expect magnetization reversal to be the time-limiting process, so that switching rates in the $\mathrm{kHz}-\mathrm{MHz}$ region should become feasible in this way. Such a device should enable highly sensitive, differential (lock-in) measurements. One could extend the photon energy range of this method to about $180 \mathrm{eV}$ [25] by using the $4 d \rightarrow 4 f$ resonances of heavier lanthanide elements. A similar scheme was proposed by Goedkoop et al. at the $M_{4,5}$ edges for the production of CP light. 31.

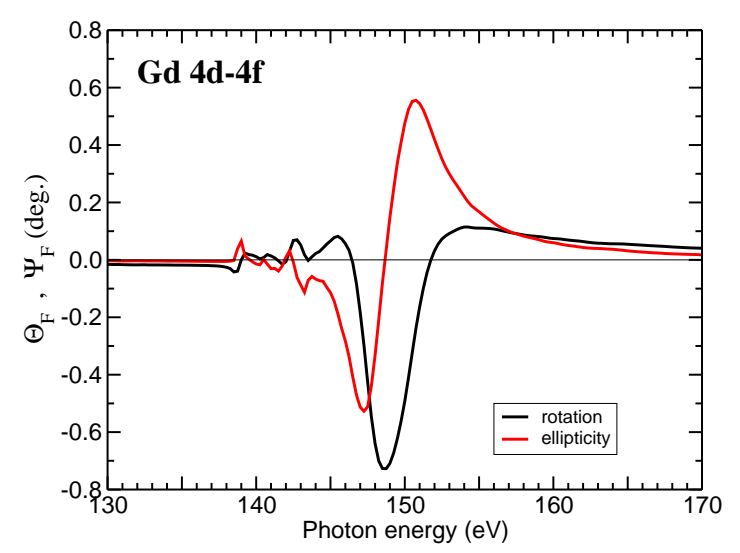

Fig. 3 Calculated Faraday rotation and ellipticity for $1 \mathrm{ML}$ $(0.3 \mathrm{~nm}) \mathrm{Gd}$ in the region of the $4 d \rightarrow 4 f$ absorption threshold.

\subsection{Dichroic reflectivity}

As a second application of the magneto-optical constants, Fig. 4(b) shows calculated reflectivity spectra of a longitudinally magnetized Gd film in the region of the $M_{4,5}$ threshold for CP incident light. The reflectivity was calculated using the Fresnel equations in the Jones matrix formalism. It includes longitudinal MO effects in the non-diagonal elements which connect the $s$ and $p$ components for transmision and reflectivity at interfaces of magnetized media. 32,33 We consider the reflected wave to be formed by the coherent superposition of the amplitudes along two different light paths, as illustrated in the insert of Fig. 4(c): (0) represents the reflection at the vacuum/Gd interface, and (1) comprises transmission through this interface, propagation in the Gd film (which includes absorption and Faraday effect), reflection at the $\mathrm{Gd} / \mathrm{W}(110)$ surface, propagation back through the film, and transmission through the $\mathrm{Gd} /$ vacuum interface. Higher-order paths that include multiple reflections inside the film are found to contribute negligibly to the reflected intensity. In the calculation, we employed the MO constants for $\mathrm{Gd}$ at the $M_{4,5}$ thresholds shown in Fig. 2 together with the values for the $\mathrm{W}$ substrate taken from Ref. 25.

Figure 4(a) shows experimental reflectivity spectra of a Gd film on W(110). The film thickness is $d=11 \pm 1 \mathrm{~nm}$ and the light incidence angle is $\theta=10 \pm 1^{\circ}$. The experimental spectra are well reproduced by the calculation shown in Fig. 4(b), including all the fine structure. The best agreement between calculated and experimental spectra was achieved by setting $d=12 \mathrm{~nm}$ and $\theta=11^{\circ}$ in the calculation. The experimental reflectivity $\mathrm{R}$ was quantified by normalizing to the intensity of the 'direct' beam, measured with the diode. Considering the uncertainties in detector position and the simplicity of the calculation (for example, no roughness was considered), the agreement of calculated with measured intensities is quite satisfactory.

It is instructive to separate the contributions of the light paths 0 and 1 shown in Fig 4(c). While the reflected intensity at the $M_{4,5}$ maxima is practically given by the reflectivity at the Gd surface alone, it is in their low-energy tails where the contribution from light paths inside the film becomes largest. Due to reduced absorption in the flanks, the light penetration length $\mu^{-1}$ is long enough for beam 1 to return to the film surface with appreciable intensity. At the high-energy side of the maxima the real part of the refractive index $(1-\delta)$ is smaller than 1 [see Fig[2(c)], i.e. the light arriving from the vacuum side passes into an optically less dense medium, so that we expect a behaviour similar to that at the glass/air interface, where total internal reflection occurs for incidence angles smaller than the critical angle. Hence, light cannot enter the Gd layer for energies right above the absorption thresholds, while at the maxima it is greatly attenuated inside the film. Despite this simple analogy to visible-light optics, the presence of absorption (imaginary parts of $n$ ) and the strong variation of the critical angle at the soft x-ray absorption maxima complicate the interpretation of $x$-ray reflectivity spectra. 

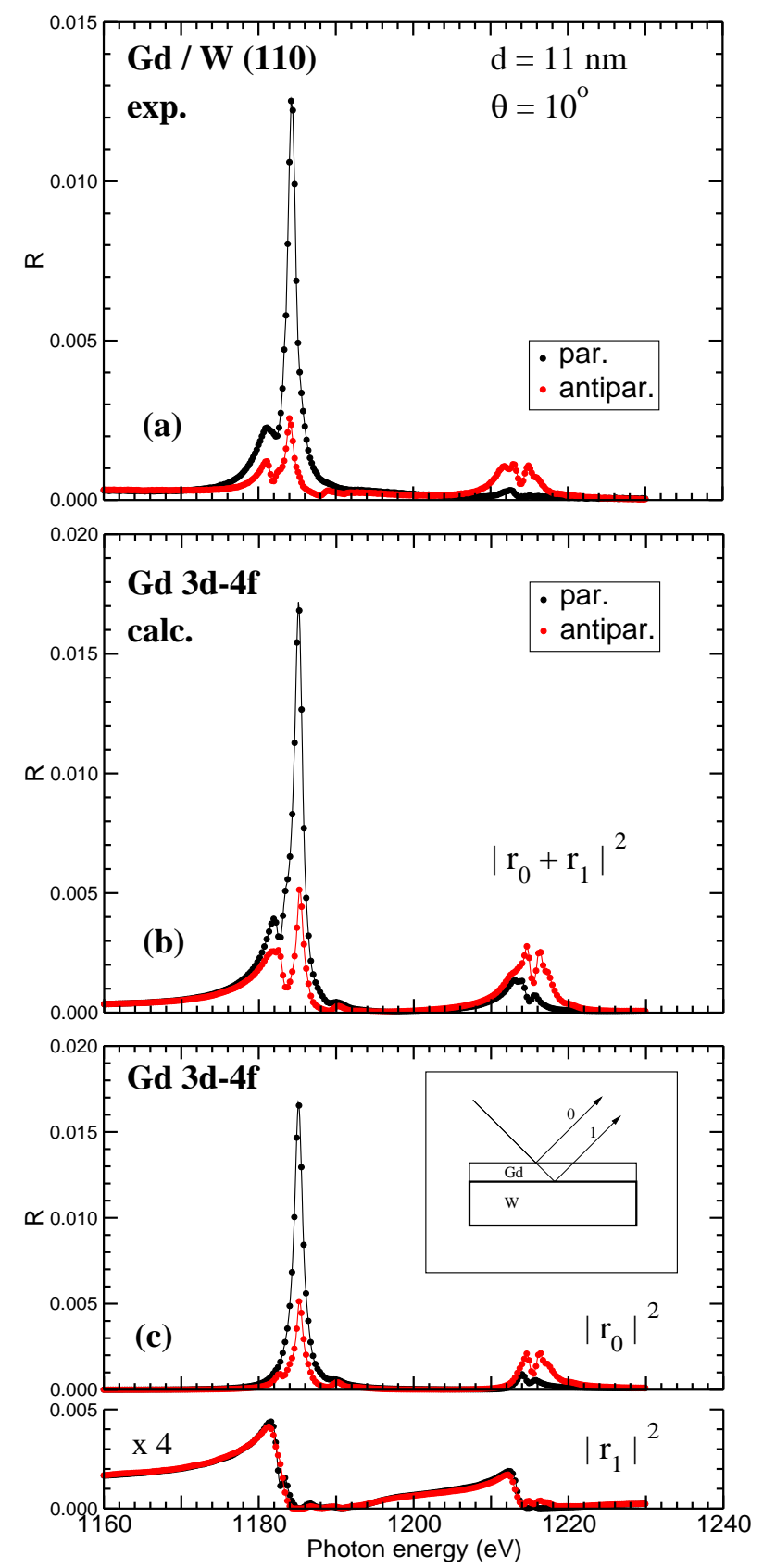

Fig. 4 Experimental (a) and calculated (b) dichroic reflectivity spectra at the $M_{4,5}$ threshold of an epitaxial Gd/W(110) film. The film thickness is $11 \mathrm{~nm}$ and the light incidence angle $10^{\circ}$. CP light was used propagating nearly parallel or antiparallel to the in-plane sample magnetization. Panel (c) shows the separate intensities of the two light paths considered in the calculations, which are schematically displayed in the insert.

\section{$4.3 \mathrm{Sm} / \mathrm{Co}$ interface}

$\mathrm{Co}_{5} \mathrm{Sm}$ is one of the hardest magnetic materials known today, much harder than pure Co metal. 34, 35. Therefore it is interesting to study the effect of Sm in the magnetic properties of very thin Co films, eventually aiming at the preparation of highly anisotropic films that can retain high ordering temperatures and high coercivities at RT even when the system dimensions approach the nanometer scale. We have performed a study of the epitaxial system $\mathrm{Sm} / \mathrm{Co}(0001)$ on $\mathrm{W}(110)$ and have found several ordered phases. [36] In the following we will concentrate on one of these phases which appears for a $\mathrm{Sm}$ coverage of about $1 \mathrm{ML}$.
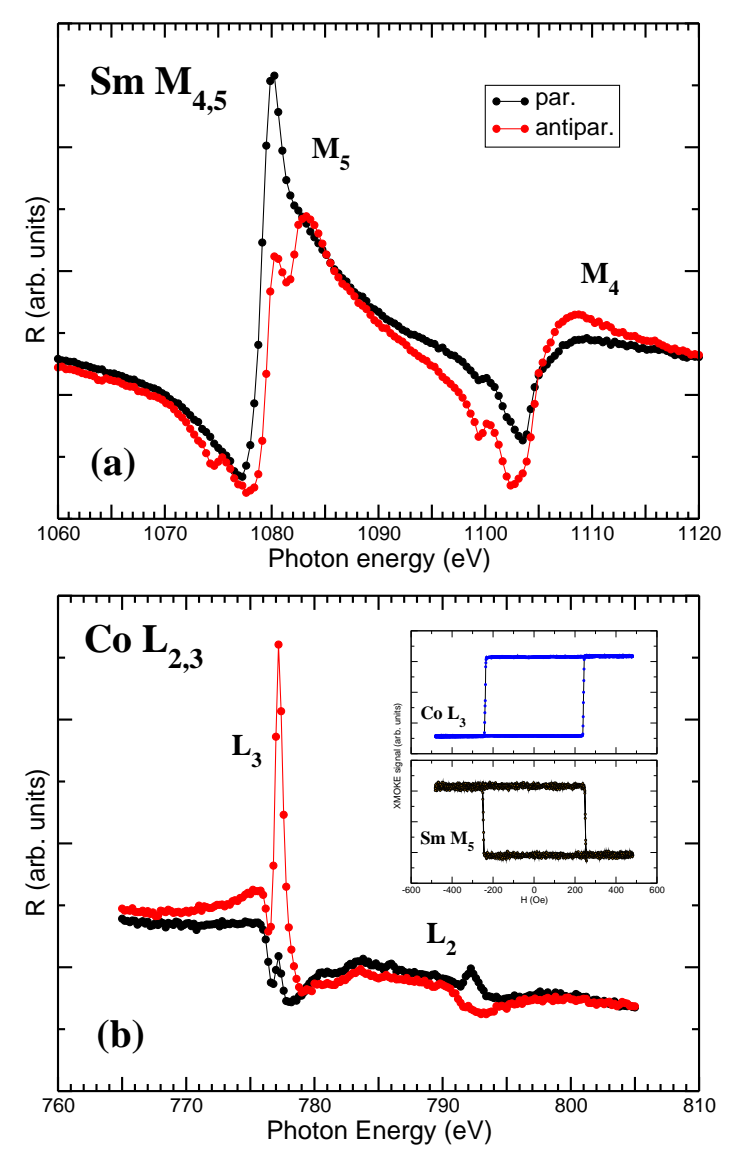

Fig. 5 Dichroic reflectivity spectra at (a) the $\mathrm{Sm}$ $M_{4,5}$ and (b) the Co $L_{2,3}$ absorption thresholds of the $\mathrm{Sm}(1 \mathrm{ML}) / \mathrm{Co}(10 \mathrm{ML}) / \mathrm{W}(110)$ structure. The insert shows element-specific hysteresis loops measured at the $\operatorname{Sm} M_{5}$ and Co $L_{3}$ reflectivity maxima with a light incidence angle of $10^{\circ}$.

Figure 5 shows soft x-ray reflectivity spectra measured on a $10 \mathrm{ML} \mathrm{Co} / \mathrm{W}(110)$ film on top of which $1 \mathrm{ML}$ $\mathrm{Sm}$ has been previously deposited. The sample was magnetized in-plane and CP light was used with the photon nearly parallel or antiparallel to the sample magnetization direction. Panel (a) corresponds to the region of the Sm $M_{4,5}$ threshold. Spectra were recorded at an incidence angle of $10^{\circ}$ with respect to the sample surface. Panel (b) shows the reflectivity spectra at the Co $L_{2,3}$ edge measured at an incidence angle of $20^{\circ}$. The spectra show sufficient magnetic contrast to allow the performance of XMOKE measurements at the Sm $M_{5}$ and Co $L_{3}$ thresholds. The result, for an incidence angle of $10^{\circ}$, is shown in the insert of Fig. 5 (b). The magnetization 
reversal process takes place homogeneously over the illuminated area, as deduced from the square shape of the hysteresis loops. Furthermore, the element-specific hysteresis of both elements reveal the same coercivity $H_{c}$ (about $250 \mathrm{Oe}$ ), showing that the film magnetization reverses simultaneously at the $\mathrm{Sm} / \mathrm{Co}$ interface and further away inside the Co film. The present Co film (10 ML) is probably too thin to allow the observation of a different magnetization reversal of the two elements that would point to a spring-magnet behaviour. 37, 38. Work is in progress aiming to prepare ordered Sm phases on top of thicker epitaxial Co films.

\section{$4.4 \mathrm{Gd} / Y / \mathrm{Tb}$ trilayers}

Exchange-coupled magnetic films through nonmagnetic spacer layers are at the heart of many modern magnetic devices such as spin valves. Here, the magnetic coupling is mediated by the conduction electrons of the spacer material and its characteristics are largely determined by their electronic structure, particularly by the topology of the Fermi surface. 39] The use of ferromagnetic rare earths (RE) as magnetic materials in exchange-coupled systems may become of interest, because the magnetic coupling inside the material is of the same type (RKKY) and of comparable strength as the interlayer coupling.

We have studied Gd / Y / Tb trilayers as a prototype interlayer exchange-coupled RE system where the two magnetic layers have widely different coercivities and ordering temperatures. While the non-spherical $4 f$ charge distribution of $\mathrm{Tb}$ couples strongly to the lattice and gives highly anisotropic, magnetically hard films, the $\mathbf{L}=0,4 f$ ground state of Gd (with a spherical charge distribution) gives much smaller coercivities. In order to ensure saturation magnetization of the $\mathrm{Tb}$ layers in our samples at low temperatures, we cooled down the samples in external fields. Results of CP x-ray reflectivity measurements of an epitaxial Gd / Y / Tb trilayer grown on W(110) are shown in Fig. 6(a). The layer thicknesses are 2.0, 1.2 and $10.0 \mathrm{~nm}$, respectively. The trilayer structure was magnetized in-plane along the $b$ axis (the easy axis of magnetization of Gd and Tb films) in a field of about 2000 Oe applied during the cool-down to $20 \mathrm{~K}$. The photon energy range comprises the $\mathrm{M}_{4,5}$ thresholds of $\mathrm{Gd}$ and $\mathrm{Tb}$. The reflectivity spectra show a sizeable MO contrast at the $\mathrm{M}_{5}$ peak of both elements. They are ferromagnetically coupled at this temperature.

XMOKE hysteresis curves recorded at the $\mathrm{M}_{5}$ maxima are shown in Fig. 6 (b) and (c) for different temperatures. The black and grey lines denote the sign of the external field applied during the cool-down of the sample to the corresponding measurement temperature. The capabibities of XMOKE as an element-specific technique are evident in this case: the exchange field between the ferromagnetic layers $\mathrm{Gd}$ and $\mathrm{Tb}$ is given directly by half of the horizontal shift of the Gd hysteresis recorded after cool-down in opposite external fields [Fig. [6) (b)]. For
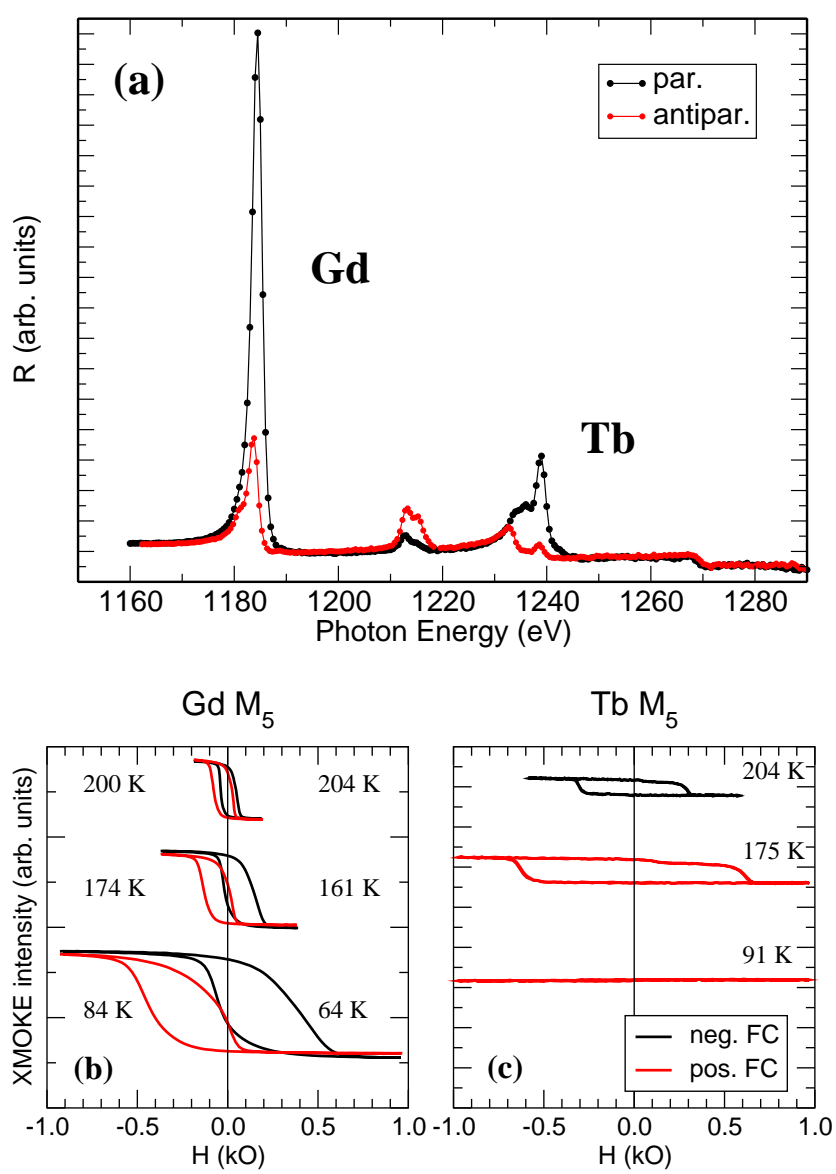

Fig. 6 (a): dichroic reflectivity spectra at the $M_{4,5}$ thresholds of $\mathrm{Gd}$ and $\mathrm{Tb}$ in a $\mathrm{Gd} / \mathrm{Y} / \mathrm{Tb}$ trilayer structure with a $1.2 \mathrm{~nm}$-thick Y spacer layer. The sample was cooled down to the measurement temperature $(20 \mathrm{~K})$ in an external field. $\mathrm{CP}$ light was incident at a grazing angle of $8^{\circ}$ nearly parallel or antiparallel to the sample magnetization. Lower panel: temperature-dependent element specific XMOKE hysteresis curves measured at the same incidence angle with the photon energy tuned to (b) the Gd and (c) the $\mathrm{Tb} M_{4,5}$ reflectivity maxima. Black and grey lines denote the sign (negative and positive, repectively) of the applied field during the cool-down of the sample to the corresponding measurement temperature.

this, it is required that the magnetically hard $\mathrm{Tb}$ layer does not reverse its magnetization within the range of the applied external field, so that the Gd hystereses can be considered as minor loops of the composite magnetic structure. That this is indeed the case is shown by the Tb hysteresis curves in Fig. 6(c). For $1.2 \mathrm{~nm}$ thickness of the Y spacer layer, the coupling between the Gd and $\mathrm{Tb}$ layers is ferromagnetic and decreases strongly with increasing temperature, as shown by Fig. 6(b). We attribute the main part of this effect to the decreasing $\mathrm{Tb}$ magnetization ( $T_{C}$ of bulk $\mathrm{Tb}$ amounts to $220 \mathrm{~K}$ ). The exchange field of 200 Oe extracted from the lowest Gd curves in Fig. 6(b) gives an exchange energy of 0.086 $\mathrm{mJ} / \mathrm{m}^{2}$ using $J=H M_{s} d_{G d}$, where $M_{s}$ and $d_{G d}$ are the 
saturation magnetization and the thickness of the $\mathrm{Gd}$ layer. This is comparable to interlayer exchange energies found in other systems. 40,41] The temperature dependence of the interlayer exchange coupling in this $\mathrm{RE}$ trilayer system is under further investigation.

\section{Summary and Outlook}

By calibrating absorption spectra we have been able to quantify the magneto-optical constants of lanthanide metals at the $4 d \rightarrow 4 f$ and $3 d \rightarrow 4 f$ excitation thresholds. We have demonstrated that the constants can be used to calculate MO properties in transmission (Faraday rotation) and reflection geometries (dichroic reflectivity spectra). Furthermore, we have demonstrated the power of x-ray magneto-optical techniques at the examples of a Sm/Co interface and exchange-coupled Gd/Y/Tb trilayer structures. X-ray MO methods are expected to play an important role in research on magnetization dynamics in the near future, particularly with the advent of free-electron lasers in the soft x-ray regime.

\section{Acknowledgments}

J.E. Prieto gratefully acknowledges financial support from the Alexander-von-Humboldt Stiftung and the Spanish MEC (grant No. EX2001 11808094). The authors thank F. Senf, R. Follath and H.-Ch. Mertins for experimental help at BESSY. This work was financially supported by the German BMBF under Contract No. 05 KS1 KEC/2.

\section{References}

1. D. Mergel, H. Heitmann, and P. Hansen. Phys. Rev. B, 47:882, 1993.

2. Z. Q. Qiu and S. D. Bader. Rev. Sci. Instrum., 71:1243, 2000 .

3. D. S. Bloomberg and G. A. N. Connell. In C. D. Mee and E. D. Daniel, editors, Magnetic Recording Handbook: Technology and Applications. McGraw-Hill, New York, 1990.

4. A. Hubert and R. Schäfer. Magnetic Domains. Springer, Berlin, Heidelberg, 1998.

5. J. S. Jiang, Eric E. Fullerton, M. Grimsditch, C. H. Sowers, and S. D. Bader. J. Appl. Phys., 83:6238, 1998.

6. S. A. Majetich and Y. Jin. Science, page 470, 1999.

7. G. A. Prince. Science, page 1660, 1998.

8. C.-C. Kao, C. T. Chen, E. D. Johnson, J. B. Hastings, H. J. Lin, G. H. Ho, G. Meigs, J. M. Brot, S. L. Hulbert, Y. U. Idzerda, and C. Vettier. Phys. Rev. B, 50:9599, 1994.

9. J. M. Tonnerre, L. Sève, A. Barbara-Dechelette, F. Bartolomé, D. Raoux, V. Chakarian, C. C. Kao, H. Fischer, S. Andrieu, and O. Fruchart. J. Appl. Phys., 83:6293, 1998.

10. J. Geissler, E. Goering, M. Justen, F. Weigand, G. Schütz, J. Langer, D. Schmitz, H. Maletta, and R. Mattheis. Phys. Rev. B, 65:020405, 2001.
11. S. A. Stepanov and S. K. Sinha. Phys. Rev. B, 61:15302, 2000.

12. M. Sacchi, C. F. Hague, L. Pasquali, A. Mirone, J.-M. Mariot, P. Isberg, E. M. Gullikson, and J. H. Underwood. Phys. Rev. Lett., 81:1521, 1998.

13. V. Chakarian, Y. U. Idzerda, and C. T. Chen. Phys. Rev. $B, 57: 5312,1998$.

14. J. B. Kortright and S.-K. Kim. Phys. Rev. B, 62:12216, 2000.

15. H.-Ch. Mertins, F. Schäfers, X. Le Cann, A. Gaupp, and W. Gudat. Phys. Rev. B, 61:R874, 2000.

16. J. Kuneš, P. M. Oppeneer, H.-Ch. Mertins, F. Schäfers, A. Gaupp, W. Gudat, and P. Novák. Phys. Rev. B, 64:174417, 2001.

17. H.-Ch. Mertins, D. Abramsohn, A. Gaupp, F. Schäfers, W. Gudat, O. Zaharko, H. Grimmer, and P. M. Oppeneer. Phys. Rev. B, 66:184404, 2002.

18. M. R. Weiss, R. Follath, K. J. S. Sawhney, F. Senf, J. Bahrdt, W. Frentrup, A. Gaupp, S. Sasaki, M. Scheer, H.-Ch. Mertins, D. Abramsohn, F. Schäfers, W. Kuch, and W. Mahler. Nucl. Instrum. Meth. Phys. Res. A, 467:449, 2001.

19. G. van der Laan, E. Arenholz, Z. Hu, A. Bauer, E. Weschke, Ch. Schüßler-Langeheine, E. Navas, A. Mühlig, G. Kaindl, J. B. Goedkoop, and N. B. Brookes. Phys. Rev. B, 59:8835, 1999.

20. Z. Hu, K. Starke, G. van der Laan, E. Navas, A. Bauer, E. Weschke, Ch. Schüßler-Langeheine, E. Arenholz, A. Mühlig, G. Kaindl, J. B. Goedkoop, and N. B. Brookes. Phys. Rev. B, 59:9737, 1999.

21. K. Starke, E. Navas, E. Arenholz, Z. Hu, L. Baumgarten, G. van der Laan, C.-T. Chen, and G. Kaindl. Phys. Rev. $B, 55: 2672,1997$.

22. F. Heigl, O. Krupin, G. Kaindl, and K. Starke. Rev. Sci. Instrum., 73:369, 2002.

23. K. Starke. Magnetic Dichroism in Core-Level Photoemission. Springer, Berlin, 2000.

24. U. Stetter, M. Farle, K. Baberschke, and W. G. Clark. Phys. Rev. B, 45:503, 1992.

25. B. L. Henke, E. M. Gullikson, and J. C. Davis. At. Data Nucl. Data Tables, 54:180, 1993. www-cxro. lbl. gov/optical_constants.

26. R. Nakajima, J. Stöhr, and Y. U. Idzerda. Phys. Rev. B, 59:6421, 1999.

27. G. van der Laan and B. T. Thole. J. Electron Spectrosc. Relat. Phenomen., 46:123, 1988.

28. J. E. Prieto, F Heigl, O. Krupin, G. Kaindl, and K Starke. Phys. Rev. B, 68:134453, 2003.

29. J. B. Goedkoop, B. T. Thole, G. van der Laan, G. A. Sawatzky, F. M. F. de Groot, and J. C. Fuggle. Phys. Rev. B, 37:2086, 1988.

30. J. E. Prieto, F Heigl, O. Krupin, G. Kaindl, and K Starke. Phys. Rev. B, 66:172408, 2002.

31. J. B. Goedkoop, J. C. Fuggle, B. T. Thole, G. van der Laan, and G. A. Sawatzky. Nucl. Instrum. Meth. Phys. Res. A, 273:429, 1988.

32. A. K. Zvezdin and V. A. Kotov. Modern Magnetooptics and Magnetooptical Materials. Institute of Physics Publishing, Bristol, 1997.

33. P. M. Oppeneer. In K. H. J. Buschow, editor, Handbook of Magnetic Materials, volume 13, page 229. NorthHolland, Amsterdam, 2001. 
34. J. F. Herbst. Rev. Mod. Phys., 63:819, 1991.

35. K. H. J. Buschow. Rep. Prog. Phys., 54:1123, 1991.

36. J. E. Prieto, O. Krupin, K. Döbrich, G. Kaindl, and K. Starke. To be published.

37. E. E. Fullerton, J. S. Jiang, C. H. Sowers, J. E. Pearson, and S. D. Bader. Appl. Phys. Lett., 72:380, 1998.

38. E. E. Fullerton, J. S. Jiang, M. Grimsditch, C. H. Sowers, and S. D. Bader. Phys. Rev. B, 58:12193, 1998.

39. P. Bruno and C. Chappert. Phys. Rev. B, 46:261, 1992.

40. Z. Celinski, B. Heinrich, and J. F. Cochran. J. Magn. Magn. Mater., 145:L1, 1995.

41. S. T. Purcell, W. Folkerts, M. T. Johnson, N. W. E. McGee, K. Jager, J. aan de Stegge, W. B. Zeper, W. Hoving, and P. Grünberg. Phys. Rev. Lett., 67:903, 1991. 